\title{
Arabidopsis AtERF15 positively regulates immunity against Pseudomonas syringae pv. tomato DC3000 and Botrytis cinerea
}

\author{
Huijuan Zhang, Lei Huang, Yi Dai, Shixia Liu, Yongbo Hong, Limei Tian, Lihong Huang, \\ Zhongye Cao, Dayong Li and Fengming Song*
}

National Key Laboratory for Rice Biology, Institute of Biotechnology, Zhejiang University, Hangzhou, China

\section{OPEN ACCESS}

Edited by:

Stéphane Hacquard, Max Planck Institute for Plant Breeding Research, Germany

Reviewed by:

Nico Tintor,

Max Planck Institute for Plant Breeding Research, Germany

Rainer Birkenbihl,

Max Planck Institute for Plant Breeding Research, Germany

*Correspondence: Fengming Song

National Key Laboratory for Rice Biology, Institute of Biotechnology,

Zhejiang University,

Hangzhou 310058, China

fmsong@zju.edu.cn

Specialty section:

This article was submitted to Plant Biotic Interactions, a section of the journal Frontiers in Plant Science

Received: 01 July 2015

Accepted: 20 August 2015 Published: 04 September 2015

Citation:

Zhang H, Huang L, Dai Y, Liu S, Hong Y, Tian L, Huang L, Cao Z, Li D and Song $F$ (2015) Arabidopsis AtERF15 positively regulates immunity against Pseudomonas syringae $\mathrm{pv}$. tomato DC3000 and Botrytis cinerea.

Front. Plant Sci. 6:686. doi: 10.3389/fpls.2015.00686
Upon pathogen infection, activation of immune response requires effective transcriptional reprogramming that regulates inducible expression of a large set of defense genes. A number of ethylene-responsive factor transcription factors have been shown to play critical roles in regulating immune responses in plants. In the present study, we explored the functions of Arabidopsis AtERF15 in immune responses against Pseudomonas syringae pv. tomato (Pst) DC3000, a (hemi)biotrophic bacterial pathogen, and Botrytis cinerea, a necrotrophic fungal pathogen. Expression of AtERF15 was induced by infection of Pst DC3000 and B. cinerea and by treatments with salicylic acid (SA) and methyl jasmonate. Biochemical assays demonstrated that AtERF15 is a nucleus-localized transcription activator. The AtERF15-overexpressing (AtERF15OE) plants displayed enhanced resistance while the AtERF15-RNAi plants exhibited decreased resistance against Pst DC3000 and B. cinerea. Meanwhile, Pst DC3000- or B. cinerea-induced expression of defense genes was upregulated in AtERF15-OE plants but downregulated in AtERF15-RNAi plants, as compared to the expression in wild type plants. In response to infection with $B$. cinerea, the AtERF15-OE plants accumulated less reactive oxygen species (ROS) while the AtERF15-RNAi plants accumulated more ROS. The flg22- and chitin-induced oxidative burst was abolished and expression levels of the pattern-triggered immunity-responsive genes AtFRK1 and AtWRKY53 were suppressed in AtER15-RNAi plants upon treatment with flg22 or chitin. Furthermore, SA-induced defense response was also partially impaired in the AtERF15-RNAi plants. These data demonstrate that AtERF15 is a positive regulator of multiple layers of the immune responses in Arabidopsis.

Keywords: Arabidopsis thaliana, ethylene-responsive factor (ERF) transcription factors, immune response, pattern-triggered immunity, Pseudomonas syringae pv. tomato DC3000, Botrytis cinerea

\section{Introduction}

Plants have evolved to possess a sophisticated innate immune system to defend themselves against pathogen attack during their lifespan (Boller and He, 2009; Dodds and Rathjen, 2010; Schwessinger and Ronald, 2012; Fu and Dong, 2013). To date, two types of innate immune responses that are timely activated and precisely regulated by different types of pathogens have been recognized in 
plants and studied extensively for their molecular, genetic and biochemical mechanisms. Pathogen-associated molecular pattern (PAMP)-triggered immunity (PTI) is often activated by PAMPs such as flagellin, EF-Tu and chitin, most of which are common structural components of microbes (Zhang and Zhou, 2010; Bernoux et al., 2011; Segonzac and Zipfel, 2011); whereas effectortriggered immunity is modulated by recognition of pathogenderived specific avirulence effectors by plant $\mathrm{R}$ proteins (Cui et al., 2009; Bigeard et al., 2015). In addition to the innate immunity, plants have also developed to possess several forms of inducible immunity that becomes activated upon pathogen infection or treatment of elicitors. Systemic acquired resistance (SAR) and induced systemic resistance (ISR), are the two types of inducible immunity that is activated by different stimuli through distinct signaling pathways (Grant and Lamb, 2006; Van Wees et al., 2008; Shoresh et al., 2010; Fu and Dong, 2013). Once the pathogenderived PAMPs or effectors and elicitors are perceived (Boller and He, 2009), a battery of immune responses is often activated by a network of defense hormone-mediated signaling pathways (Pieterse et al., 2009), which ultimately lead to transcriptional reprogramming that coordinately regulates expression of a large set of genes. The transcription reprogramming is the consequence of the concerted action of a range of transcription factors (TFs), which function directly or indirectly to deploy their activity in proper ways. TFs are divided into diverse families according to conserved structural domains, which are involved in DNA binding activity and numerous TFs belonging to the AP2/ERF, NAC, WRKY, and bZIP families have been implicated in plant immune responses against diverse pathogens (Gutterson and Reuber, 2004; Alves et al., 2013; Nuruzzaman et al., 2013; Seo et al., 2015).

The AP2/ERF superfamily is a large plant-specific TF family and consists of approximately 147 members in the Arabidopsis, of which 122 are the ERF proteins (Sakuma et al., 2002; Gutterson and Reuber, 2004; Nakano et al., 2006). The ERF proteins contain a highly conserved DNA-binding domain, which is consisted of 58 or 59 amino acid residues (Ohme-Takagi and Shinshi, 1995). Many of them have been shown to bind specifically to GCC box, a core sequence essential for activation of expression of defense genes (Ohme-Takagi and Shinshi, 1995; Büttner and Singh, 1997; Zhou et al., 1997; Solano et al., 1998). The ERFs can act as transcription activators or repressors in regulating the expression of defense genes. For example, the Arabidopsis AtERF1, AtERF2, and AtERF5 are activators while AtERF3, AtERF4, and AtERF7 act as repressors (Fujimoto et al., 2000).

The 122 Arabidopsis AtERF proteins have been divided into 12 major groups on the basis of the type of AP2 domain (Nakano et al., 2006) and many of them have not been defined for their biological functions yet. Recent studies using loss-of-function and gain-of-function Arabidopsis mutants have demonstrated that a number of the ERFs in B3 group, consisting of 17 members, play critical roles in regulating defense responses against pathogens. For example, it was shown that AtERF92 (AtERF1), AtERF100 (AtERF-1), AtERF101 (AtERF2), AtERF97 (AtERF14) and AtERF94 (ORA59) are responsive transcriptionally to pathogens and defense signaling molecules such as salicylic acid (SA), jasmonic acid (JA), and ethylene (ET) (Oñate-Sánchez and
Singh, 2002; McGrath et al., 2005; Oñate-Sánchez et al., 2007; Pré et al., 2008). It was found that overexpression (OE) of AtERF1, AtERF2, AtERF5, AtERF6, AtERF14, or ORA59 conferred an increased resistance against several pathogenic fungi including Botrytis cinerea through activating the expression of defenserelated genes such as AtPDF1.2 and ChiB (Solano et al., 1998; Berrocal-Lobo et al., 2002; Brown et al., 2003; Lorenzo et al., 2003; McGrath et al., 2005; Oñate-Sánchez et al., 2007; Pré et al., 2008; Moffat et al., 2012). The functions of ERF1, AtERF5, AtERF6, AtERF14, and ORA59 have been shown to act as regulators of the JA/ET-mediated signaling pathway (Lorenzo et al., 2003; Berrocal-Lobo and Molina, 2004; Oñate-Sánchez et al., 2007; Pré et al., 2008; Moffat et al., 2012). More recently, it was found that phosphorylation of AtERF6 by MPK3/MPK6 can increase the AtERF6 stability and thus constitutively activates defense genes including AtPDF1.1 and AtPDF1.2 and provides increased resistance against $B$. cinerea (Meng et al., 2013). In addition, AtERF5 was shown to be involved in the chitin-induced innate immunity response (Son et al., 2012). Therefore, 7 out of 17 B3 group members in the ERF family play important roles in regulating defense response against pathogens. However, the function of the other B3 group members in plant defense response remains unknown.

AtERF15, a member of the B3 group in the AtERF family, was recently shown to function as a positive regulator of abscisic acid (ABA)-mediated abiotic stress response (Lee et al., 2015). In this study, we explored the function of AtERF15 in immune response against Pseudomonas syringae pv. tomato (Pst) DC3000 and B. cinerea by analyzing the disease phenotypes and defense response in AtERF15-overexpressing (AtERF15-OE) and AtERF15-RNAi lines. Our data revealed that constitutive OE of AtERF15 conferred an increased resistance while RNAi-mediated suppression of AtERF15 led to decreased resistance against Pst DC3000 and B. cinerea. Furthermore, suppression of AtERF15 attenuated the flg22- or chitin-induced PTI response and partially impaired the SA-induced resistance. Our data demonstrate that AtERF15 is a positive regulator of multiple layers of the immune responses in Arabidopsis.

\section{Materials and Methods}

\section{Plant Growth and Treatment}

Arabidopsis thaliana ecotype Col-0 and transgenic plants were cultivated in a mixture of vermiculite: plant ash: perlite $(6: 2: 1)$ in a growth room with a rhythm of $16 \mathrm{~h}$ light/ $8 \mathrm{~h}$ dark. For treatments with signaling hormones, $1 \mathrm{mM} \mathrm{SA}$ and $100 \mu \mathrm{M}$ methyl jasmonate (MeJA) in $0.1 \%$ ethanol were sprayed evenly on the leaves of 4-week-old plants. Mock controls were set by spraying a group of plants with similar volume of $0.1 \%$ ethanol.

\section{Generation and Characterization of Transgenic Lines}

The open reading frame (ORF) of AtERF15 was obtained by RT-PCR using a pair of gene-specific primers AtERF15-orf1F (5'-ATG GAA TAT TCC CAA TCT- $\left.3^{\prime}\right)$ and AtERF15orf-1R (5'-TCA ACA TGA GCT CAT AAG-3') and cloned 
into pMD19-T vector, yielding plasmid pMD19-AtERF15. After confirmation by sequencing, plasmid pMD19-AtERF15 was used for all experiments. To construct the OE plasmid, the AtERF15 ORF was amplified from pMD19-AtERF15 using a pair of primers AtERF15-orf-2F (5'-AGT GGA TCC ATG GAA TAT TCC CAA TCT-3', a BamHI site underlined) and AtERF15-orf-2R (5'-AGA GGG CCC TCA ACA TGA GCT CAT AAG - $3^{\prime}$, a ApaI site underlined), and cloned into binary vector pCAMBIA 99-1 under control of the CaMV 35S promoter, yielding pCAMBIA991-AtERF15. To construct the AtERF15 RNAi plasmid, a fragment of 237 bp in size was amplified from pMD19-AtERF15 using a pair of primers AtERF15-Ri-1F (5' AGA TTAATTAA CCATGG GAT AAC AAA AAG AAA AGA AAA AGA G-3', PacI/NcoI sites underlined) and AtERF15Ri-1R (5'-GCG GGATCC GGCGCGCC TAA TCA TCA TCT CCG GTG ACT CAA A-3', BamHI/AscI sites underlined) and cloned into vector pGSA1165 under control of the CaMV 35 S promoter, pGSA1165-AtERF15. Arabidopsis transformation was carried out by the floral dip method (Clough and Bent, 1998). Single-copy homozygous lines were selected according to the segregation ratios (hygromycin-rsesistant:hygromycinsensitive $=3: 1$ ) on selective $1 / 2 \mathrm{MS}$ medium containing $50 \mu \mathrm{g} / \mathrm{ml}$ hygromycin.

\section{Pathogen Inoculation and Disease Assays}

Pseudomonas syringae $p v$. tomato DC3000 was cultivated in King's B (KB) liquid medium with $25 \mu \mathrm{g} / \mathrm{ml}$ rifampicin at $28^{\circ} \mathrm{C}$ in a shaker to $\mathrm{OD}_{600}=0.7 \sim 1.0$. Bacterial inoculum was prepared in $10 \mathrm{mM} \mathrm{MgCl} 2$ and adjusted to $\mathrm{OD}_{600}=0.002$. Inoculation was done using 1-ml syringes without needles by infiltrating bacterial suspension into the leaf blade or by infiltrating with similar volume of $10 \mathrm{mM} \mathrm{MgCl} 2$ solution as mock-inoculation controls. The inoculated plants were covered with a transparent plastic film to keep high humidity and photos were taken 4 days after inoculation. For the determination of bacteria population, leaf disks from six independent plants were first sterilized with $70 \%$ ethanol for $10 \mathrm{~s}$, homogenized in $200 \mu \mathrm{l} 10 \mathrm{mM} \mathrm{MgCl}_{2}$ and diluted to the proper concentration and plated on KB agar plates supplemented with $100 \mu \mathrm{g} / \mathrm{ml}$ rifampicin.

Botrytis cinerea strain BO5.10 (provided by Dr. Tesfaye Mengiste, Purdue University, USA) (Veronese et al., 2006) was cultivated on $2 \times \mathrm{V} 8$ agar medium (36\% V8 juice, $0.2 \%$ $\mathrm{CaCO}_{3}$, and $2 \%$ agar) for 10 days. Spores were collected in $1 \%$ maltose buffer to prepare the inoculum and adjusted to a final concentration of $2 \times 10^{5}$ spores $/ \mathrm{ml}$. Inoculation was carried out by spraying the spore suspension until the leaves were covered with tiny moisture (Wang et al., 2009). Chlorophyll contents were measured as described previously (Veronese et al., 2006; Wang et al., 2009) and shown as percentages of that in the corresponding mock-inoculated plants. The completely rotten plants were recorded as died plants. In planta fungal growth was estimated by analyzing the transcript levels of $B$. cinerea BcActinA gene using a pair of primers BcActinA-1F $\left(5^{\prime}\right.$-ACT CAT ATG TTG GAG ATG AAG CGC AA-3') and BcActinA-1R (5'-AAT GTT ACC ATA CAA ATC CTT ACG GAC A-3'). The Arabidopsis actin gene (AtActin) was used as an internal control and the primers were AtActin-1F (5'-GGC GAT GAA GCT CAA
TCC AAA CG-3') and AtActin-1R (5'-GGT CAC GAC CAG CAA GAT CAA GAC G-3').

\section{Subcellular Localization Assays}

The AtERF15 ORF was amplified from pMD19-AtERF15 using a pair of gene-specific primers AtERF15-s-1F (5'-GCG TCTAGA ATG GAA TAT TCC CAA TCT-3', a XbaI site underlined) and AtERF15-s-1R (5'-ATA CCCGGG TCA ACA TGA GCT CAT AAG-3', a SmaI site underlined) and inserted into vector pFGCEGFP, yielding plasmid pFGC-GFP-AtERF15. This plasmid and the pFGC-EGFP empty vector were electroporated into Agrobacterium tumefacies GV3101 and the acquired agrobacteria were injected into leaves of 4-week-old Nicotiana benthamiana plants expressing a red nuclear marker protein RFP-H2B (Chakrabarty et al., 2007) using 1-ml needless syringes. After agroinfiltration, the plants were grown in a growth room under $25^{\circ} \mathrm{C}$ for $24 \mathrm{~h}$. GFP fluorescence signals were excited at $488 \mathrm{~nm}$ and detected using a 500-530 $\mathrm{nm}$ emission filter preformed with confocal laser scanning microscope (Zeiss LSM 510 META).

\section{Transactivation Activity Assays}

The AtERF15 ORF was amplified from pMD19-AtERF15 using a pair of gene-specific primers AtERF15-y-1F (5'-AGT GTCGAC ATG GAA TAT TCC CAA TCT-3', a SalI site underlined) and AtERF15-y-1R (5'-GCG CTGCAG TCA ACA TGA GCT CAT AAG-3', a Pst I site underlined) and constructed into vector pBD-GAL4Cam, yielding plasmid pBD-AtERF15. Plasmid pBD-AtERF15, pBD-GAL4 (a positive control) and pBD empty vector (a negative control) were transformed into yeast strain AH109. The transformed yeasts were plated on deficient medium $\left(S D / \operatorname{Trp}^{-} \mathrm{His}^{-}\right)$with $\mathrm{x}$ - $\alpha$-gal and incubated for 3 days at $30^{\circ} \mathrm{C}$. Transactivation activity of the fused proteins was evaluated based on the emergence of blue pigments.

\section{RNA Extraction and qRT-PCR Analysis of Gene Expression}

Total RNA was extracted by Trizol regent (TaKaRa, Dalian, China) following the manufacturer's instructions and the firststrand cDNA was obtained using PrimeScript RT regent kit (TaKaRa, Dalian, China). The qPCR reaction was done on a CFX96 real-time PCR system (BioRad, Hercules, CA, USA) in $25 \mu \mathrm{L}$ reactions containing $12.5 \mu \mathrm{L}$ SYBR Premix Ex TaqTM (TaKaRa, Dalian, China), $0.1 \mathrm{mg}$ cDNA and $7.5 \mathrm{pmol}$ of each gene-specific primer. Relative gene expression levels were calculated using $2^{-\Delta \Delta \mathrm{CT}}$ method with three independent biological replicates. Primers used in qRT-PCR were AtPR1-q-F, 5'-TCG TCT TTG TAG CTC TTG TAG GTG-3'; AtPR1-q-R, 5' TAG ATT CTC GTA ATC TCA GCT CT-3'; AtPR5-q-F, 5'-ATG GCA AAT ATC TCC AGT ATT CAC A-3'; AtPR5-q-R, 5'-ATG TCG GGG CAA GCC GCG TTG AGG-3'; AtPDF1.2-q-F, 5'GCTA AGT TTG CTT CCA TCA TCA CCC TT-3'; AtPDF1.2q-R, 5'-AAC ATG GGA CGT AAC AGA TAC ACTTGT G-3'; AtActin-q-1F, 5'-GGC GAT GAA GCT CAA TCC AAA CG-3'; AtActin-q-1R, 5'-GGT CAC GAC CAG CAA GAT CAA GAC G-3'. AtNPR1-q-1F, 5'-CAC TAT GGC GGT TGA ATG TA3'; AtNPR1-q-1R, 5'-GGG AGG AAC ATC TCT AGG AA-3'; 
AtERF15-q-1F, 5'-AAC GGC GAC GTT TCT AAC TCC GAA-3'; AtERF15-q-1R, 5'-GCT TTG TCA AAT GTC CCG AGC CAA3'; AtFRK1-F, 5' -GCC AAC GGA GAC ATT AGA G-3'; AtFRK1R, 5'-CCA TAA CGA CCT GAC TCA TC-3'; AtWRKY53-F, $5^{\prime}$-CAC CAG AGT CAA ACC AGC CAT TAC-3'; AtWRKY53-R, $5^{\prime}$-CTT TAC CAT CAT CAA GCC CAT CGG-3'.

\section{In situ Detection of ROS and Measurement of Oxidative Burst}

In situ detection of superoxide anion and $\mathrm{H}_{2} \mathrm{O}_{2}$ was carried out using nitroblue tetrazolium (NBT) staining (Doke, 1983) and 3, 3-diaminobenzidine (DAB) staining (Thordal-Christensen et al., 1997; Li et al., 2014), respectively. Accumulation of superoxide anion and $\mathrm{H}_{2} \mathrm{O}_{2}$ in stained leaves was recorded by a digital camera. Determination of oxidative burst in leaves was carried out using a luminol-based luminescence method (Chakravarthy et al., 2010). Simply, 4-mm leaf disks from fully expanded leaves of 4-week-old plants were floated in $200 \mu \mathrm{L}$ $\mathrm{ddH}_{2} \mathrm{O}$ overnight in each well of a 96-well plate, followed by addition of $100 \mathrm{nM}$ flg22 (VI-A, Sigma, St. Louis, MO, USA) or $1 \mu \mathrm{M}$ chitin (Hepta- $N$-acetylchitoheptaose, Toronto Research Chemicals, Toronto, ON, Canada), $34 \mu \mathrm{g} / \mathrm{ml}$ luminol (Sigma, St. Louis, MO, USA) and $20 \mu \mathrm{g} / \mathrm{ml}$ horseradish peroxidase (VIA, Sigma, St. Louis, MO, USA). Addition of the same solutions without flg22 or chitin was used as controls. Luminescence was measured on a Synergy HT plate reader (Biotek Instruments, Inc. Winooski, VT, USA) and recorded every $2 \mathrm{~min}$ until $20 \mathrm{~min}$.

\section{Identifiers of the Genes Used in This Study}

AtERF1, At3g23240; AtERF2, At5g47220; AtERF3, At1g50640; AtERF4, At3g15210; AtERF5, At5g47230; AtERF6, At4g17490; AtERF7, At3g20310; AtERF15, At2g31230; AtERF94 (ORA59), At1g06160; AtERF97 (AtERF14), Atlg04370; AtFRK1: At2g19190; AtWRKY53, At4g23810; AtPR1, At2g14610; AtPR5, At1g75040; AtPDF1.2, At5g4420; AtNPR1, At1g64280; AtActin, At1g01130.

\section{Results}

\section{AtERF15 was Induced by Infection with Pathogens and Treatments of SA and MeJA}

AtERF15 encodes a 243 amino acid protein with high level of similarity to ORA59, which was shown to JA and ET signals in defense response (Pré et al., 2008), and belongs to Group IX (also known as B-3 group) of the Arabidopsis ERF family (Nakano et al., 2006). To explore its possible involvement in plant defense, we first analyzed the expression patterns of AtERF15 in response to infection by different pathogens including Pst DC3000 and B. cinerea and to treatments with defense signaling hormones such as SA and MeJA. In Pst DC3000-infected plants, the expression of AtERF15 was significantly induced at $24 \mathrm{~h}$ after inoculation, leading to a threefold increase over that in mockinoculated plants, and then decreased (Figure 1A). Similarly, the expression of AtERF15 in B. cinerea-infected plants was significantly induced at $12 \mathrm{~h}$ and maintained at relatively high
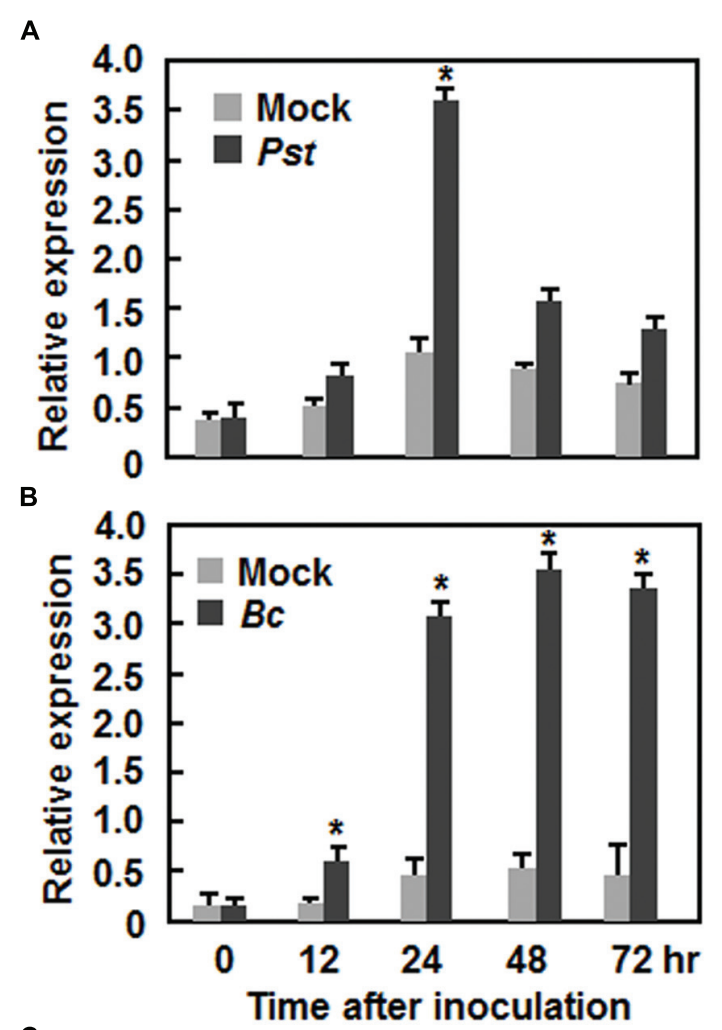

C

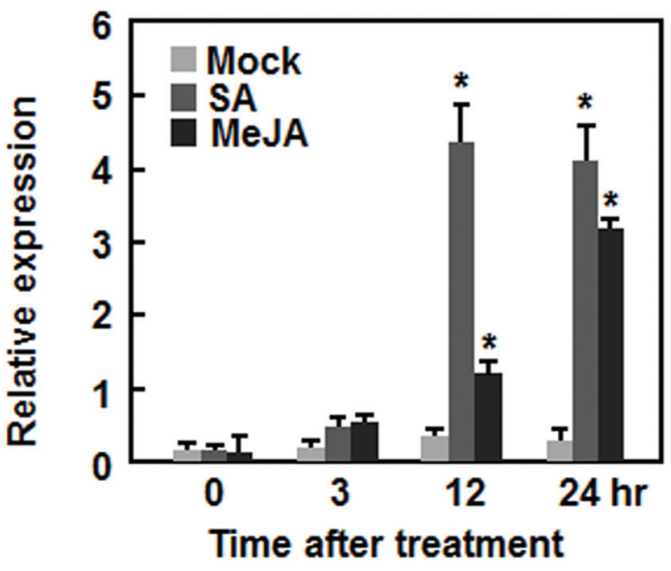

FIGURE 1 | Induction of AtERF15 by infection with Pst DC3000 and Botrytis cinerea and by treatments with salicylic acid and methyl jasmonate. (A) Induction of AtERF15 by Pst DC3000. (B) Induction of AtERF15 by $B$. cinerea. Four-week-old Col-0 plants were inoculated by vacuum infiltration with Pst DC3000 $\left(\mathrm{OD}_{600}=0.002\right)(\mathbf{A})$, by spraying with spores of $B$. cinerea $\left(2 \times 10^{5}\right.$ spores $\left./ \mathrm{ml}\right)(\mathbf{B})$, or by treatment with corresponding solution (10 $\mathrm{mM} \mathrm{MgCl}_{2}$ ) for Pst DC3000 and buffer (4\% maltose and $1 \%$ peptone) for $B$. cinerea as mock controls. (C) Induction of AtERF15 by SA and MeJA. Four-week-old Col-0 plants were treated by foliar spraying with $1 \mathrm{mM}$ salicylic acid (SA), $100 \mu \mathrm{M}$ jasmonic acid (MeJA), or $0.1 \%$ ethanol as mock controls. The inoculated or treated leaves were collected at indicated times and expression of AtERF15 was analyzed through qRT-PCR. Data were normalized with the transcript level of AtActin as an internal control and relative expression levels were shown as folds of the level of AtActin.

Data presented are the means $\pm S D$ from three independent experiments and * above the columns indicate significant differences at $p<0.05$ level between the inoculated/treated plants and mock control plants. 
levels during a period of $24-72 \mathrm{~h}$ after inoculation, giving $5 \sim$ 6 -fold increases over that in mock-inoculated plants (Figure 1B). Further, expression of AtERF15 was upregulated by SA or JA but displayed distinct patterns. The expression of AtERF15 in SA-treated plants increased significantly by 10 -fold at $12 \mathrm{~h}$ and maintained at similar level at $24 \mathrm{~h}$, as compared to mock control plants, after treatment (Figure 1C), whereas the expression of AtERF15 in JA-treated plants increased by 2.3 -fold at $12 \mathrm{~h}$ and further increased by 10 -fold at $24 \mathrm{~h}$, as compared to that in mock control plants, after treatment (Figure 1C). These results indicate that AtERF15 is responsive to pathogens and defense signaling hormones, implying an involvement of AtERF15 in defense response.

\section{AtERF15 is a Nucleus-Localized Protein with Transactivation Activity}

The biochemical features of AtERF15 were examined by analyzing the transactivation activity in yeast and subcellular localization in planta. All yeasts transformed with pBD-AtERF15, pBD-Gal4 (a positive control) or pBD empty vector (a negative control) grew well on $S D / \operatorname{Trp}^{-}$medium. However, only yeasts transformed with $\mathrm{pBD}-\mathrm{AtERF} 15$ or $\mathrm{pBD}-\mathrm{Gal} 4$ produced a blue pigment after the addition of $\mathrm{x}$ - $\alpha$-gal, indicating a $\beta$-galactosidase activity, whereas yeasts transformed with empty vector did not (Figure 2A). To examine the subcellular localization, agrobacteria carrying pFGC-EGFP::AtERF15 and pFGC-EGFP (a negative control) were injected into leaves of 4-week-old $N$. benthamiana plants harboring a red nuclear marker protein RFP-H2B (Chakrabarty et al., 2007). The GFP::AtERF15 fusion was solely and clearly localized to the nucleus, co-localized with the known nucleus marker RFP-H2B protein (Figure 2B), whereas the GFP alone distributed ubiquitously throughout the cell without specific compartmental localization (Figure 2B). These data demonstrate that AtERF15 is a nucleus-localized protein with transactivation activity.

\section{Generation and Characterization of AtERF15-Overexpressing and RNAi Lines}

Due to unavailability of appropriate T-DNA insertion mutant line for AtERF15 gene at the beginning of this study, we thus generated CaMV 35S promoter-driven OE and RNA interfering (Ri)-mediated suppression lines to determine the biological function of AtERF15 in disease resistance. Transgenic AtERF15OE and AtERF15-Ri lines were obtained through floral dip transformation and the single-copy homozygous T3 lines were obtained from T2 seeds according to the 3:1 (Hgr-resistant/Hgrsensitive) segregation ratio. Two AtERF15-OE and two AtERF15Ri lines were selected for further functional studies. qRT-PCR analysis revealed that the expression levels of AtERF15 in AtERF15-OE lines were 6.69 and 5.04 times higher than that in Col-0 plants while the expression levels of AtERF15 in AtERF15Ri lines were 19 and 26\% of the that in Col-0 plants (Figure 3A), indicating that the transgene is expressed correctly in both of the AtERF15-OE and AtERF15-Ri lines. In our experiment, no visible morphological or vegetable growth or reproductive development defect was observed in the AtERF15-OE and AtERF15-Ri plants (Figure 3B).

\section{Altered Disease Phenotypes and Defense Response in AtERF15-OE and AtERF15-Ri Plants Against Pst DC3000}

We first examined the function of AtERF15 in immunity against a bacterial pathogen by comparing disease phenotypes of the AtERF15-OE and AtERF15-Ri plants after challenging with a virulent strain of Pst DC3000. In the Col-0 plants, typical lesions were seen and bacterial growth in inoculated leaves was $1.6 \times 10^{6}$ and $2.3 \times 10^{7} \mathrm{CFU} / \mathrm{cm}^{2}$ at 2 and 4 days postinoculation (dpi), respectively (Figures 4A,B). The AtERF15-OE plants showed less disease symptoms than the Col-0 plants after inoculation with the bacterial pathogen (Figure 4A). Similarly, bacterial growth in infected leaves of the AtERF15-OE-1 and AtERF15-OE-2 plants was (1.4 and 9.1) $\times 10^{5}$ and (1.6 and $11.5) \times 10^{5} \mathrm{CFU} / \mathrm{cm}^{2}$ at 2 and $4 \mathrm{dpi}$, respectively, resulting in $10 \sim 20$ folds of decrease in bacterial growth relative to the Col- 0 plants (Figure 4B). By contrast, the AtERF15-Ri plants developed more severe disease symptoms than the Col-0 plants, forming larger chlorotic lesions on the inoculated leaves (Figure 4A). The bacterial growth in inoculated leaves of the AtERF15-Ri-1 and AtERF15-Ri-2 plants was $2.9 \times 10^{7}$ and $7.4 \times 10^{8} \mathrm{CFU} / \mathrm{cm}^{2}$ and $4.1 \times 10^{7}$ and $9.6 \times 10^{8} \mathrm{CFU} / \mathrm{cm}^{2}$ at 2 and $4 \mathrm{dpi}$, respectively,
A

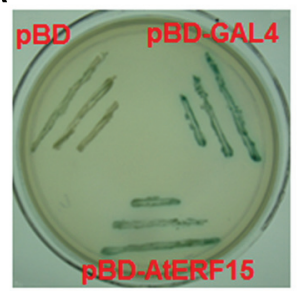

B

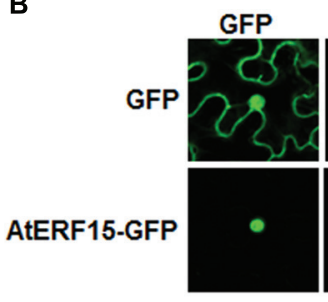

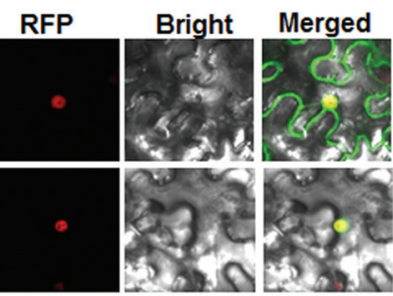

FIGURE 2 | Transactivation activity and nuclear localization of AtERF15 protein. (A) AtERF15 has transactivation activity. Yeast cells carrying pBD-AtERF15, pBD-GAL4 (a positive control) or pBD empty vector (a negative control) were grown on $\mathrm{SD}^{\mathrm{Trp}}{ }^{-}$plates for 3 days at $30^{\circ} \mathrm{C}$ and activity of $\beta$-galactosidase was detected by addition of $x$ - $\alpha$-gal. (B) AtERF15 is localized in nucleus. Agrobacteria carrying pFGC-Egfp-AtERF15 or pFGC-Egfp empty vector were infiltrated into leaves of Nicotinana benthamiana plants expressing a red nucleus marker protein RFP-H2B and leaf samples were collected at $24 \mathrm{~h}$ after infiltration for observation under a confocal laser scanning microscope. Images were taken in dark field for GFP and RFP, bright field for cell morphology and in combination, respectively. 


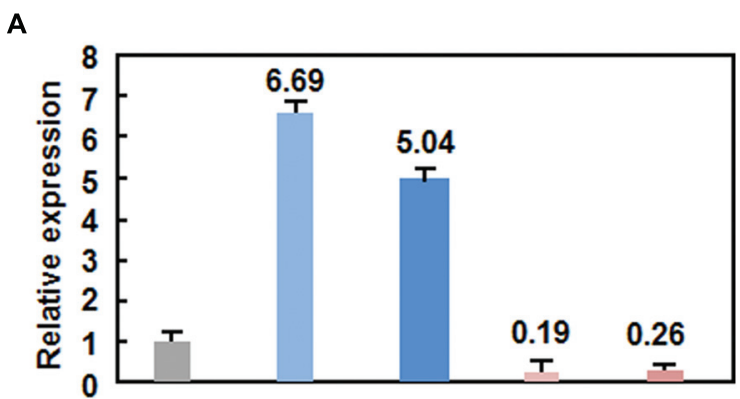

B

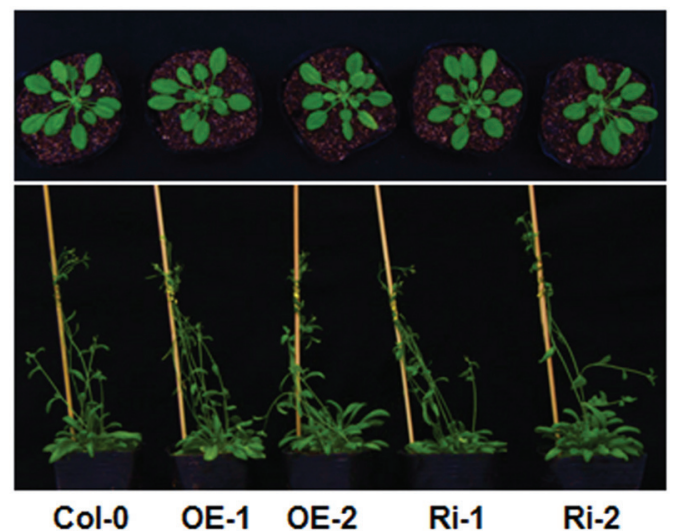

FIGURE 3 | No defects in growth and reproduction of the AtERF15-overexpressing (OE) and AtERF15-RNAi (Ri) plants.

(A) Expression levels of AtERF15 in AtERF15-OE and AtERF15-Ri lines analyzed by qRT-PCR. (B) Morphological comparison of the AtERF15-OE and AtERF15-Ri plants with the Col-0 plants. Top panel, 4-week-old plants; bottom panel, 6-week-old plants.

resulting in significant increases of $18 \sim 41$ folds in bacterial growth, compared with those in Col-0 plants (Figure 4B). These results indicate that OE of AtERF15 resulted in a decreased in planta bacterial growth and less disease symptoms and while suppression of AtERF15 led to an increased in planta bacterial growth and severe disease symptoms.

To examine whether the altered disease phenotypes observed in AtERF15-OE and AtERF15-Ri plants are linked to changes in the regulation of defense responses due to OE or suppression of AtERF15, we measured the expression levels of some defenserelated genes (i.e., AtPR1, AtPR5, and AtPDF1.2) in the Col-0,x AtERF15-OE and AtERF15-Ri plants after infection of Pst DC3000. Without infection, the expression levels of the selected defense genes in the AtERF15-OE and AtERF15-Ri plants were similar to those in the Col-0 plants (Figure 4C), indicating that altered expression of AtERF15 did not affect the expression of defense-related genes. The expression of AtPR1 and AtPR5 in the Col-0 plant was significantly induced by Pst DC3000 at $24 \mathrm{~h}$ after inoculation, as compared with those in the mockinoculated plants (Figure 4C). When compared with those in the Col-0 plants, the pathogen-induced expression of AtPR1 and AtPR5 showed 2- or 3-fold increase in the AtERF15OE plants but had 2 or 3 -fold reduction in the AtERF15-Ri plants at $24 \mathrm{~h}$ after inoculation with Pst DC3000 (Figure 4C).
A

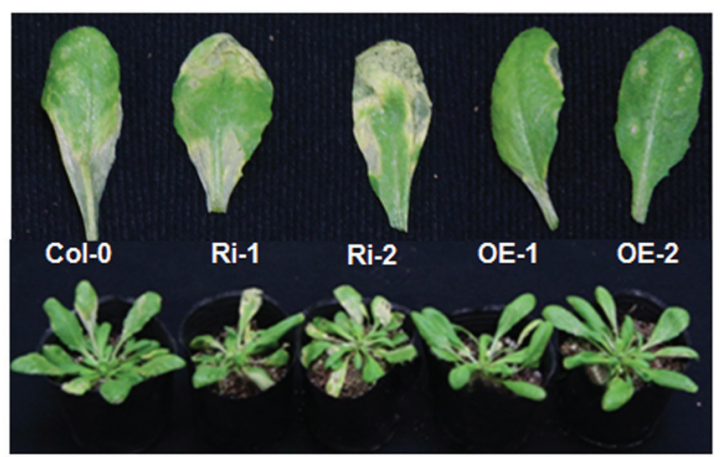

B
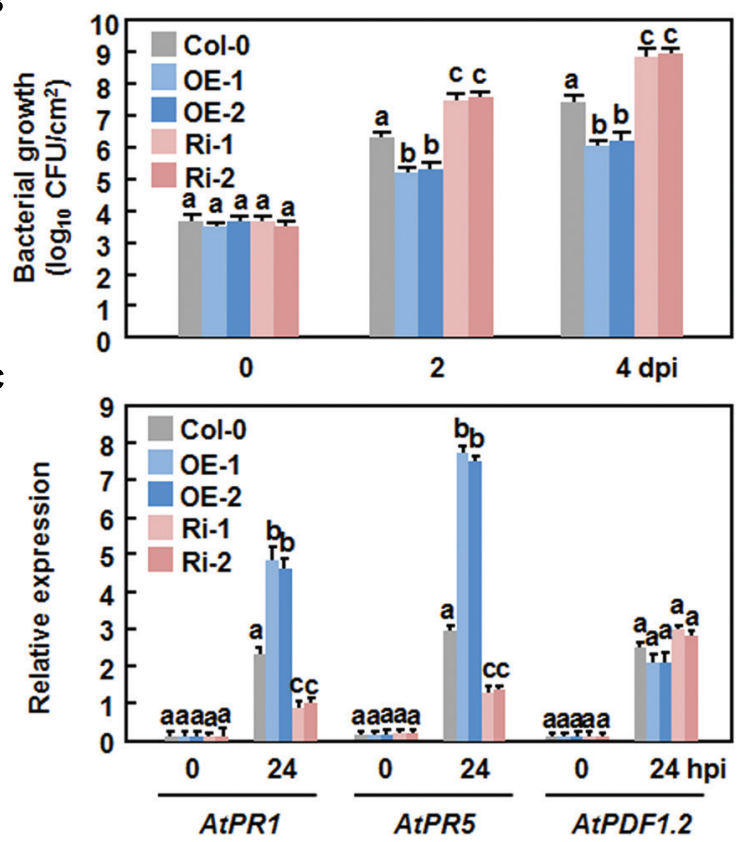

FIGURE 4 | Altered disease responses of the AtERF15-OE and AtERF15-Ri plants to Pst DC3000. (A) Disease symptom on Col-0, AtERF15-OE and AtERF15-Ri plants. Four-week-old plants were infiltrated with Pst DC3000 $\left(\mathrm{OD}_{600}=0.002\right)$. Photos were taken 4 days after inoculation. (B) Bacterial growth in infected leaves of Col-0, AtERF15-OE and AtERF15-Ri plants. Leaf samples were collected at 0, 2, and 4 days after inoculation and bacterial growth in CFU $/ \mathrm{cm}^{2}$ leaf area was shown. (C) Expression of defense genes in Col-0, AtERF15-OE and AtERF15-Ri plants after inoculation with Pst DC3000. Expression of defense genes was analyzed by qRT-PCR and the transcript data obtained were normalized with the value of a reference AtActin gene. Relative expression levels of the defense genes were expressed as folds of the AtActin gene. Data presented are the means $\pm S D$ from three independent experiments and and different letters above the columns indicate significant differences at $p<0.05$ level between the AtERF15-OE or AtERF15-Ri and Col-0 plants.

The expression of AtPDF1.2 in the Col-0, AtERF15-OE and AtERF15-Ri plants was slightly affected in the same way by infection of Pst DC3000 (Figure 4C). These data indicate the OE or suppression of $A t E R F 15$ can prime or attenuate the activation of defense response in Arabidopsis plants upon infection with Pst DC3000, linking to the altered immunity in the AtERF15-OE and AtEF15-Ri plants. 


\section{Altered Disease Phenotypes and Defense Response in AtERF15-OE and AtERF15-Ri Plants Against $B$. cinerea}

We next explored the possible role of AtERF15 in immunity against $B$. cinerea, a typical necrotrophic fungal pathogen with distinct infection style from that of Pst DC3000, by comparing the disease phenotype of the AtERF15-OE and AtERF15-Ri plants. In the Col-0 plants, typical necrotic and chlorotic lesions of disease symptom were seen at $4 \mathrm{dpi}$; however, these lesions were not merged into large necrotic area during the 8-day experimental period (Figure 5A). Relative chlorophyll contents in leaves of the inoculated plants were reduced by $\sim 18 \%$ at $6 \mathrm{dpi}$ (Figure 5B) and $\sim 14 \%$ of the inoculated plants were completely died at 12 dpi (Figure 5C). In contrast, the AtERF15-OE plants displayed less disease symptom and no significant lesion was seen even at $8 \mathrm{dpi}$ (Figure 5A). The relative chlorophyll content in leaves of the AtERF15-OE-1 and AtERF15-OE-2 plants was slightly reduced by $\sim 15 \%$ (Figure $5 \mathrm{~B}$ ) and only $7 \%$ of the inoculated plants were completely died at 12 dpi (Figure 5C). However, the AtERF15-Ri plants exhibited severe susceptibility to B. cinerea. At $8 \mathrm{dpi}$, large necrotic and chlorotic lesions appeared on leaves of the AtERF15-Ri-1 and AtERF15-Ri-2 plants, leading to extensive tissue damage (Figure 5A). The relative chlorophyll contents in leaves of the AtERF15-Ri-1 and AtERF15-Ri-2 plants were significantly decreased, leading to a reduction of $\sim 40 \%$ (Figure 5B), and $\sim 30 \%$ of the inoculated plants were completely died at 12 dpi (Figure 5C). Furthermore, in planta growth of $B$. cinerea in inoculated plants was also measured to confirm the disease phenotypes observed in AtERF15-OE and AtERF15Ri plants. To do this, the accumulation of the $B$. cinerea actin A $(B c A c t i n A)$ gene transcript, which is indicative of the rate of in planta fungal growth (Benito et al., 1998), was determined and compared among the Col-0, AtERF15-OE and AtERF15-Ri plants using qRT-PCR. Accumulation of the BcActinA transcript in the Col-0, AtERF15-OE and AtERF15-Ri plants was correlated well with the disease symptoms (Figure 5D). In the Col-0 plants, the abundance of the BcActinA transcript increased as infection advanced (Figure 5D). In the AtERF15-OE plants, the BcActinA transcript increased slower and accumulated to significant lower levels, resulting in reduction of $\sim 50$ and $\sim 65 \%$ at 24 and $48 \mathrm{hpi}$, respectively, as compared to those in the Col-0 plants (Figure 5D). In contrast, significantly higher levels of the BcActinA transcript accumulated in the AtERF15$\mathrm{Ri}$ plants, leading to increases of $\sim 90$ and $\sim 105 \%$ at 24 and $48 \mathrm{hpi}$, respectively, as compared to those in the Col-0 plants (Figure 5D). Taken together, these data indicate that $\mathrm{OE}$ of AtERF15 led to decreased susceptibility while suppression of AtERF15 resulted in increased susceptibility to $B$. cinerea, as confirmed by both the disease phenotypes and in planta fungal growth.

Generation and accumulation of reactive oxygen species (ROS) have been implicated in susceptible responses against necrotrophic fungi including $B$. cinerea (Mengiste, 2012). We thus compared the production of ROS in the Col-0, AtERF15$\mathrm{OE}$ and AtERF15-Ri plants after B. cinerea inoculation by in situ NTB staining of superoxide anion (Doke, 1983) and

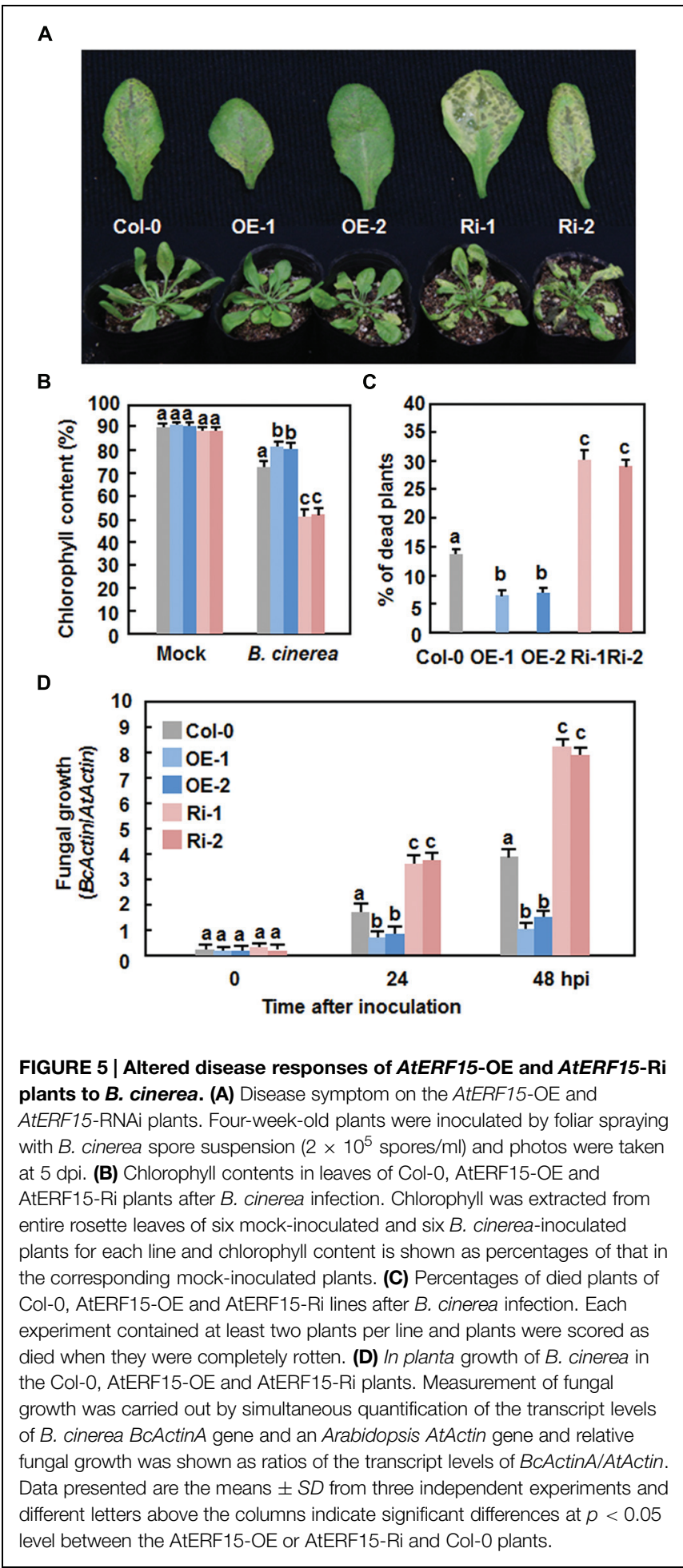

DAB staining of $\mathrm{H}_{2} \mathrm{O}_{2}$ (Thordal-Christensen et al., 1997). In mock-inoculated plants, no significant accumulation of superoxide anion and $\mathrm{H}_{2} \mathrm{O}_{2}$ was seen in the Col-0, AtERF15$\mathrm{OE}$ and AtERF15-Ri plants (Figure 6A). Upon infection of $B$. cinerea, dramatic accumulation of superoxide anion and $\mathrm{H}_{2} \mathrm{O}_{2}$ was shown in leaves of the Col-0 plants at $48 \mathrm{~h}$ after 


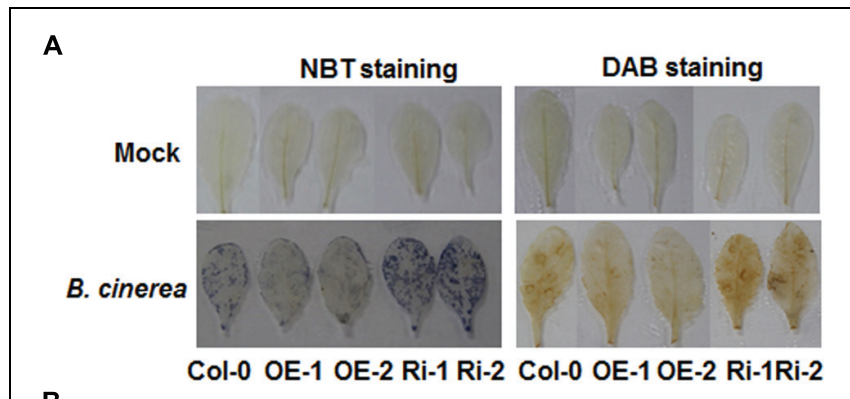

B

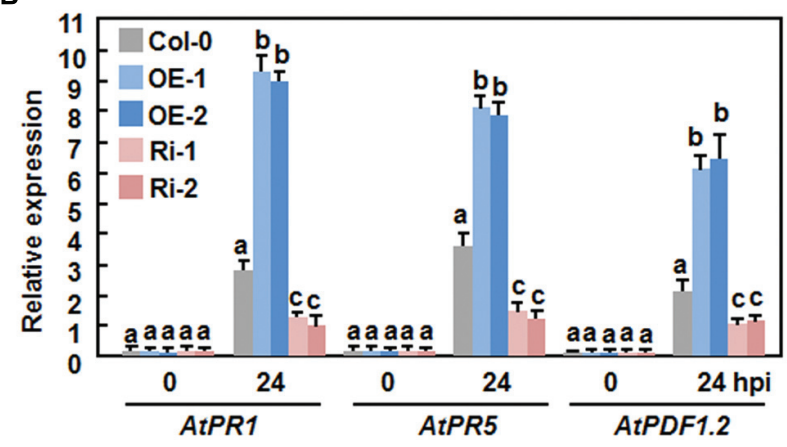

FIGURE 6 | Altered ROS accumulation and defense gene expression in AtERF15-OE and AtERF15-Ri plants after infection with B.cinerea.

Four-week-old plants were inoculated by foliar spraying with $B$. cinerea spore suspension or mock inoculated with similar volume of solution and leaf samples were taken $24 \mathrm{~h}$ after inoculation. (A) In situ detection of accumulation of superoxide anion (left) and $\mathrm{H}_{2} \mathrm{O}_{2}$ (right) after infection of B. cinerea. Accumulation of superoxide anion and $\mathrm{H}_{2} \mathrm{O}_{2}$ in leaves was detected by NBT staining and DAB staining, respectively. (B) Expression of defense genes after $B$. cinerea infection. Expression of defense genes was analyzed by GRT-PCR and the transcript data were normalized with the value of a reference AtActin gene. Relative expression levels of the defense genes were expressed as folds of the AtActin gene. Data presented are the means $\pm S D$ from three independent experiments and different letters above the columns indicate significant differences at $p<0.05$ level between the AtERF15-OE or AtERF15-Ri and Col-0 plants.

inoculation (Figure 6A). When compared with those of the Col-0 plants, however, less accumulation of superoxide anion and $\mathrm{H}_{2} \mathrm{O}_{2}$ was detected in leaves of the AtERF15-OE plants, whereas more accumulation of superoxide anion and $\mathrm{H}_{2} \mathrm{O}_{2}$ was seen in leaves of the AtERF15-Ri plants (Figure 6A). These results indicate that $\mathrm{OE}$ or suppression of AtERF15 affected the generation and accumulation of ROS upon infection of B. cinerea.

We further compared the expression changes of some defenserelated genes in Col-0, AtERF15-OE and AtERF15-Ri plants after infection of $B$. cinerea to explore the relationship between the altered immunity and transcriptional defense response. Without infection of $B$. cinerea $(0 \mathrm{hr})$, the expression levels of these selected defense genes in the AtERF15-OE and AtERF15-Ri plants were comparable to those in the Col0 plants (Figure 6B), indicating that $\mathrm{OE}$ or suppression of AtERF15 did not affect the defense response in plants. Upon infection of B. cinerea, the expression of AtPR1, AtPR5, and $A t P D F 1.2$ in the Col-0 plant was significantly upregulated, as compared with those in the mock-inoculated plants, at
$24 \mathrm{~h}$ after inoculation with Pst DC3000 (Figure 6B). When compared with those in the Col-0 plants, the $B$. cinerea-induced expression of AtPR1, AtPR5, and AtPDF1.2 showed threefold increase for AtPR1 and AtPDF1.2 and twofold increase for AtPR5 in the AtERF15-OE plants but decreased to half of that in the AtERF15-Ri plants at $24 \mathrm{~h}$ after inoculation (Figure 6B). These results suggest the $\mathrm{OE}$ or suppression of AtERF15 can affect the activation of defense response in Arabidopsis plants upon infection of $B$. cinerea and the changes in the expression of the selected defense-related genes is correlated to the altered immunity in the AtERF15-OE and AtEF15-Ri plants.

\section{Attenuation of flg22 and Chitin-Triggered PTI Responses in AtERF15-Ri Plants}

The fact that suppression of AtERF15 decreased immunity against Pst DC3000 (Figure 4) led us to hypothesize that AtERF15 may have a function in regulation of PTI response. To test this hypothesis, we first compared the PAMP-induced ROS burst, an early PTI response (Kadota et al., 2014), between the Col-0 and AtERF15-Ri plants after treatment with flg22 or chitin, two typical PAMPs derived from bacteria and fungi, respectively. In these assays, no constitutive ROS burst was detected in mock-treated Col-0 and AtERF15-Ri plants (Figures 7A,B). Significant ROS burst in leaves of the Col-0 plants was observed around 6 and $10 \mathrm{~min}$ after addition of flg22 and chitin, respectively; however, only a much reduced ROS burst was seen in leaves of the AtERF15Ri-1 and AtERF15-Ri-2 plants after addition of flg22 and chitin (Figures $7 \mathbf{A}, \mathbf{B}$ ). We next examined and compared the changes in expression of FLG22-INDUCED RECEPTOR-LIKE KINASE 1 (AtFRK1) and AtWRKY53, two well-known PTIresponsive genes (Asai et al., 2002; Singh et al., 2012), between the Col-0 and AtERF15-Ri plants after treatment with flg22 or chitin. The expression levels of AtFRK1 and AtWRKY53 were comparable between the Col-0 and AtERF15-Ri plants in mock controls (Figures 8A,B), suggesting that suppression of AtERF15 expression did not affect the expression of these two PTI-responsive genes. Treatments of the leaf disks with flg22 or chitin significantly upregulated the expression of AtFRK1 and AtWRKY53 in the Col-0 plants, leading to $>15$ folds for AtFRK1 and $>5$ folds for AtWRKY53, as compared with those in mock controls (Figures 8A,B). However, the flg22and chitin-upregulated expression of AtFRK1 and AtWRKY53 was markedly suppressed in the AtERF5-Ri plants, resulting in $50-75 \%$ of reduction as compared to those in the Col-0 plants (Figures 8A,B). These results indicate that suppression of AtERF15 attenuated the flg22- and chitin-induced PTI responses in AtERF15-Ri plants, including ROS burst and expression of PTI-responsive genes.

\section{Attenuation of SA-Induced Defense Response in AtERF15-Ri Plants}

The fact that the expression of AtERF15 was upregulated by SA led us to examine whether AtERF15 is required for SAinduced defense response. To test this hypothesis, we first assessed the SA-induced disease resistance in the AtERF15-Ri 


\section{A}

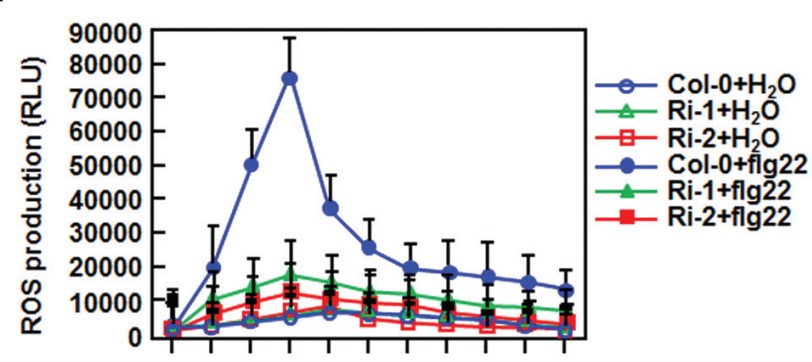

B

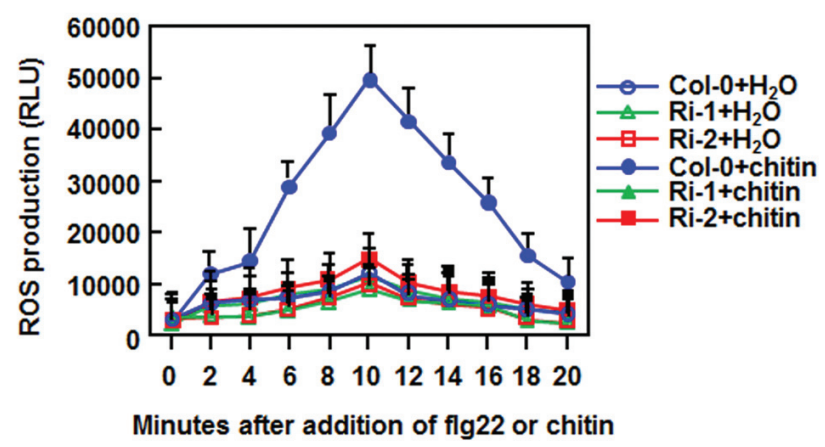

FIGURE 7 | Abolishment of flg22- and chitin-induced ROS burst in AtERF15-Ri plants. (A) Detection of flg22-induced ROS burst. (B) Detection of chitin-induced ROS burst. Leaf samples were harvested from four-week-old plants and ROS burst was measured by a luminol-based assay immediately after addition of flg22 (100 nM), chitin $(1 \mu \mathrm{M})$ or solution. Repeated experiments showed similar results.

plants by comparing disease phenotypes and bacterial growth in Col-0 and AtERF15-Ri plants after Pst DC3000 infection. The SA-treated Col-0 plants showed less severe disease symptom than the water-treated plants at $4 \mathrm{dpi}$ (Figure 9A). The SAtreated AtERF15-Ri plants showed less disease than the watertreated plants but displayed more disease than the SA-treated Col-0 plants (Figure 9A). At 3 dpi, the bacterial growth was calculated as $1.4 \times 10^{7} \mathrm{CFU} / \mathrm{cm}^{2}$ in water-treated Col-0 plants and $(2.1$ and 2.6$) \times 10^{8} \mathrm{CFU} / \mathrm{cm}^{2}$ in water-treated AtERF15-Ri plants, and as $1.1 \times 10^{6} \mathrm{CFU} / \mathrm{cm}^{2}$ in SA-treated Col-0 plants and $(3.2$ and 4.4$) \times 10^{7} \mathrm{CFU} / \mathrm{cm}^{2}$ in SA-treated AtERF15-Ri plants, respectively (Figure 9B). The bacterial population in SAtreated Col- 0 plants was reduced by 10.4 folds, whereas the bacterial populations in the SA-treated AtERF-Ri-1 and AtERFRi-2 plants were decreased by 6.5 and 5.9 folds, respectively, compared with those in water-treated Col-o and AtERF15-Ri plants (Figure 9B). We further compared the expression changes of AtPR1, a marker defense gene in SAR, and AtNPR1, a key regulatory gene critical for SAR, in Col-0 and AtERF15-Ri plants after SA treatment. In the Col-0 plants, SA significantly induced expression of AtPR1 and AtNPR1 at $24 \mathrm{~h}$ after treatment (Figure 9C). Although SA also induced expression of AtPR1 and AtNPR1 in the AtERF15-Ri plants, their expression levels were significantly reduced, leading to reduction of $40 \%$ for AtPR1 and $120 \%$ for AtNPR1, as compared with those in the Col-0 plants (Figure 9C), indicating an attenuated SA-induced defense response in AtERF15-Ri plants. Collectively, these results demonstrate that suppression of AtERF15 partially impaired the SA-induced defense responses in AtERF15-Ri plants.

\section{Discussion}

The importance of the B3 group ERFs in plant immunity has been documented by the characterization of seven members as positive or negative regulators of immune responses (Berrocal-Lobo et al., 2002; Lorenzo et al., 2003; Oñate-Sánchez et al., 2007; Pré et al., 2008; Moffat et al., 2012). In the present study, OE of AtERF15 conferred increased resistance while RNAi-mediated suppression of AtERF15 led to decreased resistance against Pst DC3000 and B. cinerea (Figures 4 and 5). Furthermore, RNAi-mediated suppression of AtERF15 also attenuated flg22- and chitininduced PTI response (Figure 7) and partially impaired the SAinduced defense response (Figure 8). These findings demonstrate that AtERF15 acts as a positive regulator of Arabidopsis immune responses against Pst DC3000 and B. cinerea, representing a hemibiotrophic bacterial pathogen and a necrotrophic fungal pathogen, respectively.

Generally, defense responses against (hemi)biotrophic pathogens such as Pst DC3000 are modulated via the SA signaling pathway, while defense responses against necrotrophic pathogens like $B$. cinerea are thought to be mediated by the JA/ET signaling pathways (Glazebrook, 2005; Grant and Jones, 2009; Verhage et al., 2010). Whereas most of the studies reported antagonistic interaction between the SA and JA/ET signaling pathways, positive cross-talks between these two defense signaling pathways do exist in response to pathogen infections (Glazebrook, 2005; Mur et al., 2006; Derksen et al., 2013). Previous studies have shown that AtERF1, AtERF5, AtERF6, AtERF14, and ORA59 in the B3 group act as regulators 

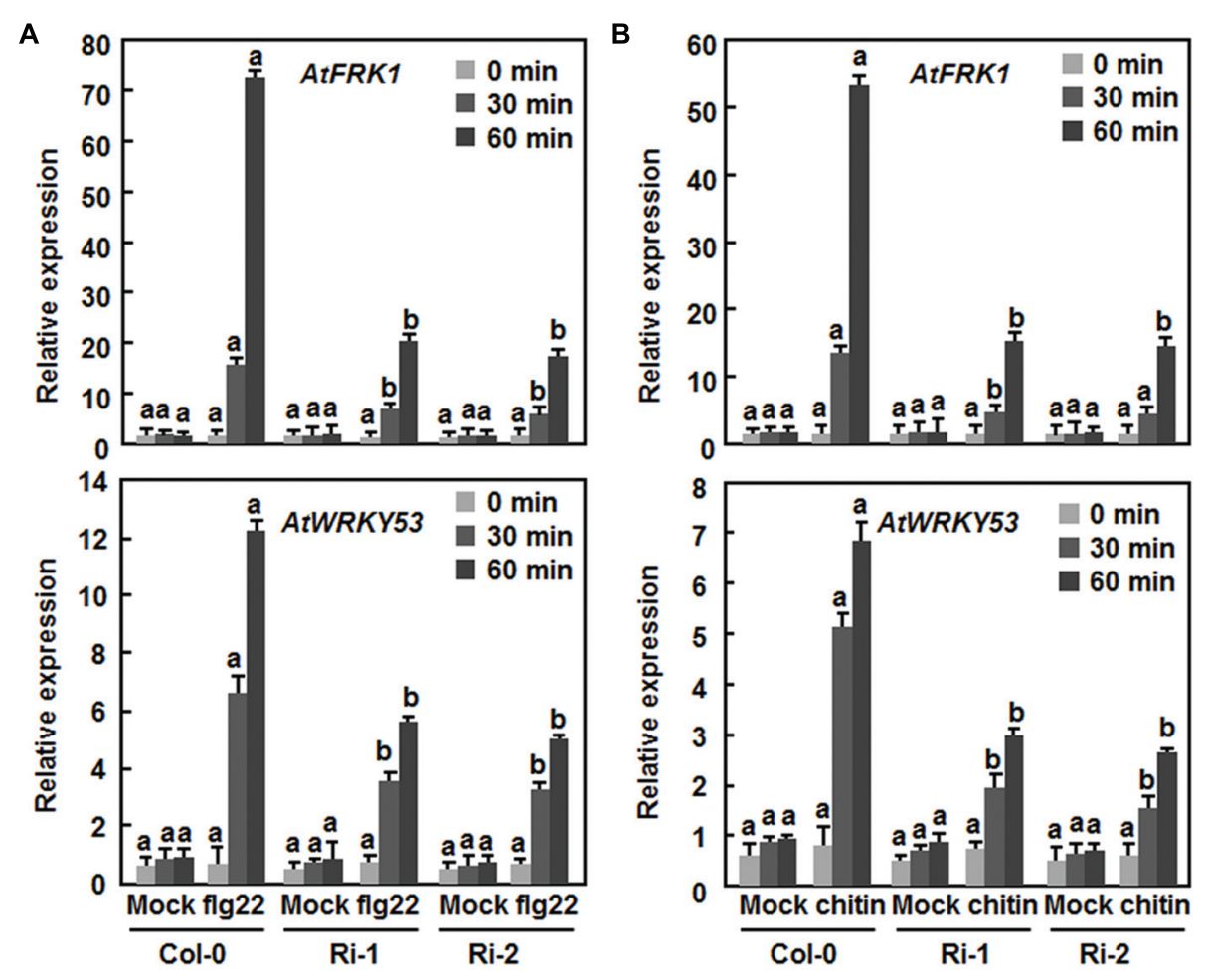

FIGURE 8 | Attenuation of flg22- and chitin-induced expression of PTI marker genes in AtERF15-Ri plants. (A) Expression of flg22-induced expression of AtFRK1 and AtWRKY53. (B) Expression of chitin-induced expression of AtFRK1 and AtWRKY53. Disks from leaves of four-week-old plants were treated with flg22 $(100 \mathrm{nM})$, chitin $(1 \mu \mathrm{M})$ or solutions as mock controls. Relative expression levels of the defense genes were expressed as folds of the AtActin gene. Data presented are the means $\pm S D$ from three independent experiments and different letters above the columns indicate significant differences at $p<0.05$ level between the AtERF15-Ri and Col-0 plants at the same time points.

of the JA/ET signaling pathway (Berrocal-Lobo et al., 2002; Lorenzo et al., 2003; Oñate-Sánchez et al., 2007; Pré et al., 2008; Moffat et al., 2012). However, our data presented in this study imply that AtERF15 may function in both of the SA and JA/ET signaling pathways. Firstly, expression of AtERF15 was induced by Pst DC3000 and B. cinerea as well as by SA and MeJA (Figure 1). ET also induced expression of AtERF15 (Oñate-Sánchez et al., 2007). Secondly, OE of AtERF15 led to increased resistance while suppression of AtERF15 resulted in decreased resistance against Pst DC3000 and B. cinerea (Figures 4 and 5). This is different from the functions of AtERF5 and AtERF6, which have positive functions in defense against $B$. cinerea but negatively regulate defense against Pst DC3000 (Moffat et al., 2012). The function of AtERF15 in defense against $B$. cinerea is supported by the patterns of pathogen-induced ROS accumulation in AtERF15-OE and AtERF15-Ri plants, which correlates with the general concept that excessive accumulation of ROS often benefits the infection by B. cinerea (Mengiste, 2012). Thirdly, expression of both SA- and JA-responsive defense genes was affected with similar patterns in AtERF15-OE and AtERF15-Ri plants by infection of Pst DC3000 and B. cinerea (Figures 4C and 6B). It was observed that $\mathrm{OE}$ of some $\mathrm{B} 3$ group members can constitutively upregulate the expression levels of some defense-related genes (Oñate-Sánchez et al., 2007; Pré et al., 2008; Moffat et al.,
2012). By contrast, OE or suppression of AtERF15 had no effect on the expression of the defense genes examined (Figures 4 and 6). The expression levels of the defense genes exhibited significant additional increases in the AtERF15-OE plants but were decreased in the AtERF15-Ri plants, as compared with those in the Col-0 plants, after pathogen infection (Figures 4 and 6). These observations indicate that AtERF15 functions as a positive regulator to prime the defense response upon pathogen infection. Furthermore, the involvement of AtERF15 in the SA signaling pathway can be further validated by the facts that the SA-induced defense response in the AtERF15-Ri plants was partially impaired (Figure 9). The observations that SA induced expression of AtERF15 (Figure 1) and the SA-induced expression of AtNPR1 in AtERF15-Ri plants was significantly suppressed (Figure 9) imply that AtERF15 may function between SA and NPR1 in the SA signaling pathway. Further analyzing the AtERF15-dependent expression patterns of AtICS1 and AtWRKY33 in AtERF15-Ri plants will clarify the mechanism whether the attenuated SA-dependent defense responses in AtERF15-Ri plants is caused by the effect of AtERF15 on the SA biosynthesis or on the later signaling. Lastly, the abolishment the flg22- and chitin-induced ROS burst (Figure 7) and the expression of PTI marker genes AtFRK1 and AtWRKY53 (Figure 8) in AtERF15-Ri plants not only demonstrates the requirement of AtERF15 in innate 
A

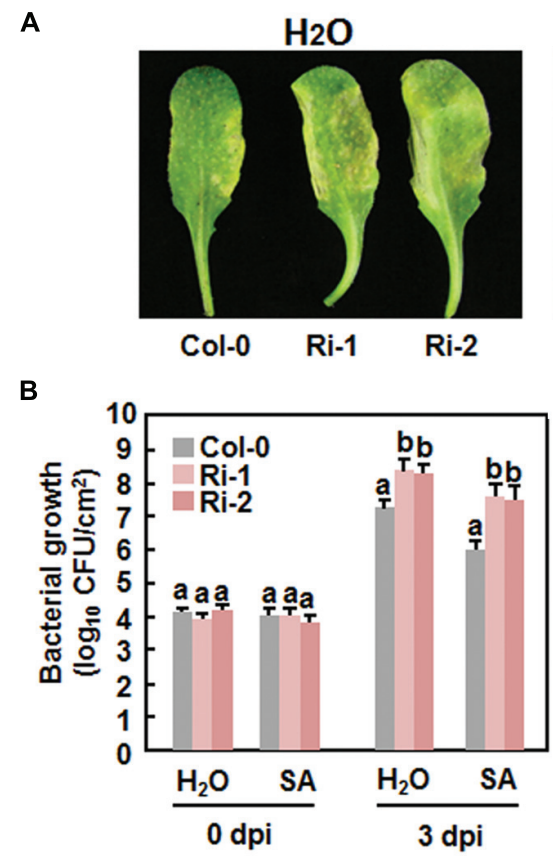

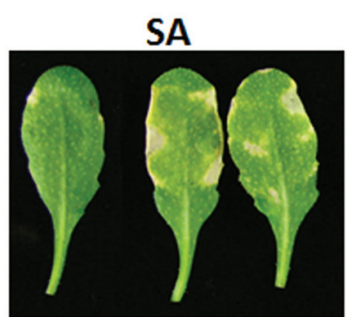

Col-0 Ri-1 Ri-2

C

FIGURE 9 | Attenuation of SA-induced defense response in AtERF15-Ri plants. (A) and (B) Attenuated SA-induced resistance against Pst DC3000 in the AtERF15-Ri plants. Four-week-old plants were treated by $1 \mathrm{mM} \mathrm{SA}$ or water and then infiltration-inoculated with $P_{s t} \mathrm{DC} 3000\left(\mathrm{OD}_{600}=0.002\right) 24 \mathrm{hr}$ later. (A) Disease symptom in representative inoculated leaves at $4 \mathrm{dpi}$ and (B) bacterial populations in inoculated leaves. (C) Expression changes of defense genes after SA treatment. Four-week-old Col-0 and AtERF15-Ri plants were treated with $1 \mathrm{mM}$ SA and expression of defense genes was analyzed using qRT-PCR. The transcript data obtained were normalized with the value of a reference AtActin gene and relative expression of the defense genes as shown as folds of the AtActin gene. Data presented are the means $\pm S D$ from three independent experiments and and different letters above the columns indicate significant differences at $p<0.05$ level between the AtERF15-Ri and Col-0 plants.

immune response but also further supports the conclusion that AtERF15 is a positive regulator of defense responses against Pst DC3000 and B. cinerea. However, the specific involvement of AtERF15 in PTI sub-branches needs to be further studied. AtERF5 was found to negatively regulate chitin signaling and defense response to Alternaria brassicicola but positively regulate SA signaling and defense response to Pst DC3000 (Son et al., 2012). Therefore, it appears that AtERF15 and AtERF5 play roles in innate immunity response with different mechanisms, although they belong to the same B3 group.

It was previously reported that AtERF14 plays a nonredundant role in defense response against Fusarium oxysporum (Oñate-Sánchez et al., 2007). In the present study, OE or RNAi-mediated suppression of AtERF15 led to clear phenotype alterations in disease resistance against Pst DC3000 and B. cinerea (Figures 4 and 5). Thus, AtERF15 and AtERF14, two closely related members in the $\mathrm{B} 3$ group (Nakano et al., 2006), function independently in Arabidopsis immune responses. However, AtERF15 showed reduced induction by ET in aterf14 mutant plants (Oñate-Sánchez et al., 2007), indicating a functional relationship between AtERF14 and AtERF15 in defense response.

The involvement of the ERF proteins in plant growth and development has been well-documented (Licausi et al., 2013). Among the three group members, OE of AtERF1, ORA59 or AtERF14 was found to cause significant growth inhibition phenotypes under normal conditions and upregulated expression of defense genes (Solano et al., 1998; Oñate-Sánchez et al., 2007; Pré et al., 2008). By contrast, we did not observed any morphological and developmental changes (Figure 3) and altered expression of defense genes (Figures 4 and 6) in the AtERF15-OE and AtERF15-Ri plants grown under normal condition. When overexpressed in Ws-0 background, the mature AtERF15-OX plants were morphologically similar to wild-type plants (Lee et al., 2015). Similar observations were also obtained for AtERF5 and AtERF6, whose OE and T-DNA insertion mutant plants are morphologically normal as the wild type plants (Son et al., 2012). Thus, it is likely that AtERF15 has limited function in growth and development.

Together with the recent finding that AtERF15 functions as a positive regulator of ABA response (Lee et al., 2015), it can be concluded that AtERF15 is a critical regulator of biotic and abiotic stress responses in Arabidopsis. Our biochemical assays revealed that AtERF15 is a nucleus-localized transcriptional activator (Figure 2) (Lee et al., 2015). It is likely that AtERF15 regulates directly or indirectly the expression of downstream target genes. This is partially supported by the observations on the altered expression patterns of some selected defense-related genes in AtERF15-OE and AtERF15-Ri plants after pathogen infection (Figures 4 and 6). On this regard, identification of downstream target genes and their functions will be helpful to elucidate the mechanism for the action of AtERF15 in regulation of biotic and 
abiotic stress responses in Arabidopsis. Furthermore, it is worth to point out that the timely dynamics of AtERF15 function seems to play roles in different aspects of immune response because AtERF15 is not only required for oxidative burst in PTI but also is involved in hormone signaling pathways, which occur later in PTI. This suggests that the AtERF15 protein can be differentially or effectively activated upon elicitation and pathogen infection.

\section{Author Contributions}

HZ, LH, YD, SL, YH, LT, LhH, ZC, and DL carried out most of the experiments. FS and $\mathrm{HZ}$ designed the experiments and wrote the paper. All authors read and approved the final manuscript.

\section{References}

Alves, M. S., Dadalto, S. P., Gonçalves, A. B., De Souza, G. B., Barros, V. A., and Fietto, L. G. (2013). Plant bZIP transcription factors responsive to pathogens: a review. Int. J. Mol. Sci. 14, 7815-7828. doi: 10.3390/ijms140 47815

Asai, T., Tena, G., Plotnikova, J., Willmann, M. R., Chiu, W. L., Gómez-Gómez, L., et al. (2002). MAP kinase signaling cascade in Arabidopsis innate immunity. Nature 415, 977-983. doi: 10.1038/415977a

Benito, E. P., ten Have, A., van't Klooster, J. W., and van Kan, J. A. L. (1998). Fungal and plant gene expression during synchronized infection of tomato leaves by Botrytis cinerea. Eur. J. Plant Pathol. 104, 207-220. doi: 10.1023/A:1008698116106

Bernoux, M., Ellis, J. G., and Dodds, P. N. (2011). New insights in plant immunity signaling activation. Curr. Opin. Plant Biol. 14, 512-518. doi: 10.1016/j.pbi.2011.05.005

Berrocal-Lobo, M., and Molina, A. (2004). Ethylene response factorl mediates Arabidopsis resistance to the soilborne fungus Fusarium oxysporum. Mol. Plant Microbe Interact. 17, 763-770. doi: 10.1094/MPMI.2004.17.7.763

Berrocal-Lobo, M., Molina, A., and Solano, R. (2002). Constitutive expression of ETHYLENE-RESPONSE-FACTOR1 in Arabidopsis confers resistance to several necrotrophic fungi. Plant J. 29, 23-32. doi: 10.1046/j.1365313x.2002.01191.x

Bigeard, J., Colcombet, J., and Hirt, H. (2015). Signaling mechanisms in pattern-triggered immunity (PTI). Mol. Plant 8, 521-539. doi: 10.1016/j.molp.2014.12.022

Boller, T., and He, S. Y. (2009). Innate immunity in plants: an arms race between pattern recognition receptors in plants and effectors in microbial pathogens. Science 324, 742-744. doi: 10.1126/science. 1171647

Brown, R. L., Kazan, K., McGrath, K. C., Maclean, D. J., and Manners, J. M. (2003). A role for the GCC-box in jasmonate-mediated activation of the PDF1.2 gene of Arabidopsis. Plant Physiol. 132, 1020-1032. doi: 10.1104/pp.102.0 17814

Büttner, M., and Singh, K. B. (1997). Arabidopsis thaliana ethylene-responsive element binding protein (AtEBP), an ethylene-inducible, GCC box DNAbinding protein interacts with an ocs element binding protein. Proc. Natl. Acad. Sci. U.S.A. 94, 5961-5966.

Chakrabarty, R., Banerjee, R., Chung, S. M., Farman, M., Citovsky, V., Hogenhout, S. A., et al. (2007). PSITE vectors for stable integration or transient expression of autofluorescent protein fusions in plants: probing Nicotiana benthamiana-virus interactions. Mol. Plant Microbe Interact. 20, 740-750. doi: 10.1094/MPMI-207-0740

Chakravarthy, S., Velasquez, A. C., Ekengren, S. K., Collmer, A., and Martin, G. B. (2010). Identification of Nicotiana benthamiana genes involved in pathogenassociated molecular pattern-triggered immunity. Mol. Plant Microbe Interact. 23, 715-726. doi: 10.1094/MPMI-23-6-0715

\section{Acknowledgments}

We are grateful to Dr. Michael Goodin (Department of Plant Pathology, University of Kentucky, USA) for providing the H2B-RFP N. benthamiana line, Dr. Yan Liang (Institute of Biotechnology, Zhejiang University) for providing samples of flg22 and chitin, and Dr. Shuqun Zhang (College of Life Sciences, Zhejiang University) for access of the Synergy HT plate reader machine in measurement of oxidative burst. This work was supported by the National High-Tech R\&D Program (No. 2012AA101505), the National Natural Science Foundation (No.31272028), the National Transgenic Major Project of China (No. 2011ZX08009-003-001), and the Research Fund for the Doctoral Program of Higher Education of China (20120101110070).

Clough, S. J., and Bent, A. F. (1998). Floral dip: a simplified method for Agrobacterium-mediated transformation of Arabidopsis thaliana. Plant J. 16, 735-743. doi: 10.1046/j.1365-313x.1998.00343.x

Cui, H., Xiang, T., and Zhou, J. M. (2009). Plant immunity: a lesson from pathogenic bacterial effector proteins. Cell. Microbiol. 11, 1453-1461. doi: 10.1111/j.1462-5822.2009.01359.x

Derksen, H., Rampitsch, C., and Daayf, F. (2013). Signaling cross-talk in plant disease resistance. Plant Sci. 207, 79-87. doi: 10.1016/j.plantsci.2013.03.004

Dodds, P. N., and Rathjen, J. P. (2010). Plant immunity: towards an integrated view of plant-pathogen interactions. Nat. Rev. Genet. 11, 539-548. doi: $10.1038 / \operatorname{nrg} 2812$

Doke, N. (1983). Involvement of superoxide anion generation in the hypersensitive response of potato tuber tissue to infection with an incompatible race of Phytophthora infestans and to the hyphal wall components. Physiol. Plant Pathol. 23, 345-357. doi: 10.1016/0048-4059(83)90019-X

Fu, Z. Q., and Dong, X. N. (2013). Systemic acquired resistance: turning local infection into global defense. Annu. Rev. Plant Biol. 64, 839-863. doi: 10.1146/annurev-arplant-042811-105606

Fujimoto, S. Y., Ohta, M., Usui, A., Shinshi, H., and Ohme-Takagi, M. (2000). Arabidopsis ethylene-responsive element binding factors act as transcriptional activators or repressors of GCC box-mediated gene expression. Plant Cell 12, 393-404. doi: 10.1105/tpc.12.3.393

Glazebrook, J. (2005). Contrasting mechanisms of defense against biotrophic and necrotrophic pathogens. Annu. Rev. Phytopathol. 43, 205-227. doi: 10.1146/annurev.phyto.43.040204.135923

Grant, M., and Lamb, C. (2006). Systemic immunity. Curr. Opin. Plant Biol. 9, 414-420. doi: 10.1016/j.pbi.2006.05.013

Grant, M. R., and Jones, J. D. (2009). Hormone (dis)harmony moulds plant health and disease. Science 324, 750-752. doi: 10.1126/science.1173771

Gutterson, N., and Reuber, T. L. (2004). Regulation of disease resistance pathways by AP2/ERF transcription factors. Curr. Opin. Plant Biol. 7, 465-471. doi: 10.1016/j.pbi.2004.04.007

Kadota, Y., Sklenar, J., Derbyshire, P., Stransfeld, L., Asai, S., Ntoukakis, V., et al. (2014). Direct regulation of the NADPH oxidase RBOHD by the PRRassociated kinase BIK1 during plant immunity. Mol. Cell 54, 43-55. doi: 10.1016/j.molcel.2014.02.021

Lee, S. B., Lee, S. J., and Kim, S. Y. (2015). AtERF15 is a positive regulator of ABA response. Plant Cell Rep. 34, 71-81. doi: 10.1007/s00299-014-1688-2

Li, X., Zhang, Y., Huang, L., Ouyang, Z., Hong, Y., Zhang, H. J., et al. (2014). Tomato SIMKK2 and SIMKK4 contribute to disease resistance against Botrytis cinerea. BMC Plant Biol. 14:166. doi: 10.1186/1471-2229-14-166

Licausi, F., Ohme-Takagi, M., and Perata, P. (2013). APETALA2/Ethylene Responsive Factor (AP2/ERF) transcription factors: mediators of stress responses and developmental programs. New Phytol. 199, 639-649. doi: 10.1111/nph.12291

Lorenzo, O., Piqueras, R., Sánchez-Serrano, J. J., and Solano, R. (2003). ETHYLENE RESPONSE FACTOR1 integrates signals from ethylene and 
jasmonate pathways in plant defense. Plant Cell 15, 165-178. doi: 10.1105/tpc.0 07468

McGrath, K. C., Dombrecht, B., Manners, J. M., Schenk, P. M., Edgar, C. I., Maclean, D. J., et al. (2005). Repressor- and activator-type ethylene response factors functioning in jasmonate signaling and disease resistance identified via a genome-wide screen of Arabidopsis transcription factor gene expression. Plant Physiol. 139, 949-959. doi: 10.1104/pp.105.068544

Meng, X., Xu, J., He, Y., Yang, K. Y., Mordorski, B., Liu, Y., et al. (2013). Phosphorylation of an ERF transcription factor by Arabidopsis MPK3/MPK6 regulates plant defense gene induction and fungal resistance. Plant Cell 25, 1126-1142. doi: 10.1105/tpc.112.109074

Mengiste, T. (2012). Plant immunity to necrotrophs. Annu. Rev. Phytopathol. 50, 267-294. doi: 10.1146/annurev-phyto-081211-172955

Moffat, C. S., Ingle, R. A., Wathugala, D. L., Saunders, N. J., Knight, H., and Knight, M. R. (2012). ERF5 and ERF6 play redundant roles as positive regulators of JA/Et-mediated defense against Botrytis cinerea in Arabidopsis. PLoS ONE 7:e35995. doi: 10.1371/journal.pone.0035995

Mur, L. A., Kenton, P., Atzorn, R., Miersch, O., and Wasternack, C. (2006). The outcomes of concentration-specific interactions between salicylate and jasmonate signaling include synergy, antagonism, and oxidative stress leading to cell death. Plant Physiol. 140, 249-262. doi: 10.1104/pp.105.072348

Nakano, T., Suzuki, K., Ohtsuki, N., Tsujimoto, Y., Fujimura, T., and Shinshi, H. (2006). Identification of genes of the plant-specific transcription-factor families cooperatively regulated by ethylene and jasmonate in Arabidopsis thaliana. J. Plant Res. 119, 407-413. doi: 10.1007/s10265-006-0287-x

Nuruzzaman, M., Sharoni, A. M., and Kikuchi, S. (2013). Roles of NAC transcription factors in the regulation of biotic and abiotic stress responses in plants. Front. Microbiol. 4:248. doi: 10.3389/fmicb.2013.00248

Ohme-Takagi, M., and Shinshi, H. (1995). Ethylene-inducible DNA binding proteins that interact with an ethylene-responsive element. Plant Cell 7, 173182. doi: 10.1105/tpc.7.2.173

Oñate-Sánchez, L., Anderson, J. P., Young, J., and Singh, K. B. (2007). AtERF14, a member of the ERF family of transcription factors, plays a nonredundant role in plant defense. Plant Physiol. 143, 400-409. doi: 10.1104/pp.106. 086637

Oñate-Sánchez, L., and Singh, K. B. (2002). Identification of Arabidopsis ethyleneresponsive element binding factors with distinct induction kinetics after pathogen infection. Plant Physiol. 128, 1313-1322. doi: 10.1104/pp.010862

Pieterse, C. M. J., Reyes, A. L., Ent, S. V. D., and Wees, S. C. M. V. (2009). Networking by small-molecule hormones in plant immunity. Nat. Chem. Biol. 5, 308-316. doi: 10.1038/nchembio.164

Pré, M., Atallah, M., Champion, A., De Vos, M., Pieterse, C. M., and Memelink, J. (2008). The AP2/ERF domain transcription factor ORA59 integrates jasmonic acid and ethylene signals in plant defense. Plant Physiol. 147, 1347-1357. doi: 10.1104/pp.108.117523

Sakuma, Y., Liu, Q., Dubouzet, J. G., Abe, H., Shinozaki, K., and YamaguchiShinozaki, K. (2002). DNA-binding specificity of the ERF/AP2 domain of Arabidopsis DREBs, transcription factors involved in dehydration- and coldinducible gene expression. Biochem. Biophys. Res. Commun. 290, 998-1009. doi: 10.1006/bbrc.2001.6299

Schwessinger, B., and Ronald, P. C. (2012). Plant innate immunity: perception of conserved microbial signatures. Annu. Rev. Plant Biol. 63, 451-482. doi: 10.1146/annurev-arplant-042811-105518

Segonzac, C., and Zipfel, C. (2011). Activation of plant patternrecognition receptors by bacteria. Curr. Opin. Microbiol. 14, 54-61. doi: 10.1016/j.mib.2010.12.005
Seo, E., Choi, D., and Choi. (2015). Functional studies of transcription factors involved in plant defenses in the genomics era. Brief. Funct. Genomics 14, 260-267. doi: 10.1093/bfgp/elv011

Shoresh, M., Harman, G. E., and Mastouri, F. (2010). Induced systemic resistance and plant responses to fungal biocontrol agents. Annu. Rev. Phytopathol. 48, 21-43. doi: 10.1146/annurev-phyto-073009-114450

Singh, P., Kuo, Y. C., Mishra, S., Tsai, C. H., Chien, C. C., Chen, C. W., et al. (2012). The lectin receptor kinase-VI.2 is required for priming and positively regulates Arabidopsis pattern-triggered immunity. Plant Cell 24, 1256-1270. doi: 10.1105/tpc.112.095778

Solano, R., Stepanova, A., Chao, Q., and Ecker, J. R. (1998). Nuclear events in ethylene signaling: a transcriptional cascade mediated by ETHYLENEINSENSITIVE3 and ETHYLENE-RESPONSE-FACTOR1. Genes Dev. 12, 3703-3714. doi: 10.1101/gad.12.23.3703

Son, G. H., Wan, J., Kim, H. J., Nguyen, X. C., Chung, W. S., Hong, J. C., et al. (2012). The Ethylene Responsive Element Binding Factor 5, ERF5, is involved in the chitin-induced innate immunity response. Mol. Plant Microbe Interact. 25, 48-60. doi: 10.1094/MPMI-06-11-0165

Thordal-Christensen, H., Zhang, Z., Wei, Y., and Collinge, D. B. (1997). Subcellular localization of $\mathrm{H} 2 \mathrm{O} 2$ in plants. $\mathrm{H} 2 \mathrm{O} 2$ accumulation in papillae and hypersensitive response during the barley-powdery mildew interaction. Plant J. $11,1187-1194$

Van Wees, S. C., Van der Ent, S., and Pieterse, C. M. (2008). Plant immune responses triggered by beneficial microbes. Curr. Opin. Plant Biol. 11, 443-448. doi: 10.1016/j.pbi.2008.05.005

Verhage, A., van Wees, S. C., and Pieterse, C. M. (2010). Plant immunity: it's the hormones talking, but what do they say? Plant Physiol. 154, 536-540. doi: 10.1104/pp.110.161570

Veronese, P., Nakagami, H., Bluhm, B., Abuqamar, S., Chen, X., Salmeron, J. et al. (2006). The membrane-anchored BOTRYTIS-INDUCED KINASE1 plays distinct roles in Arabidopsis resistance to necrotrophic and biotrophic pathogens. Plant Cell 18, 257-273. doi: 10.1105/tpc.105.0 35576

Wang, X., Basnayake, B. M., Zhang, H., Li, G., Li, W., Virk, N., et al. (2009). The Arabidopsis ATAF1, a NAC transcription factor, is a negative regulator of defense responses against necrotrophic fungal and bacterial pathogens. Mol. Plant Microbe Interact. 22, 1227-1238. doi: 10.1094/MPMI-22-1 0-1227

Zhang, J., and Zhou, J. M. (2010). Plant immunity triggered by microbial molecular signatures. Mol. Plant 3, 783-793. doi: 10.1093/mp/ssq035

Zhou, J., Tang, X., and Martin, G. B. (1997). The Pto kinase conferring resistance to tomato bacterial speck disease interacts with proteins that bind a cis-element of pathogenesis-related genes. EMBO J. 16, 3207-3218. doi: 10.1093/emboj/16.11.3207

Conflict of Interest Statement: The authors declare that the research was conducted in the absence of any commercial or financial relationships that could be construed as a potential conflict of interest.

Copyright (C) 2015 Zhang, Huang, Dai, Liu, Hong, Tian, Huang, Cao, Li and Song. This is an open-access article distributed under the terms of the Creative Commons Attribution License (CC BY). The use, distribution or reproduction in other forums is permitted, provided the original author(s) or licensor are credited and that the original publication in this journal is cited, in accordance with accepted academic practice. No use, distribution or reproduction is permitted which does not comply with these terms. 